\title{
Off -Pump Coronary Artery Bypass Grafting Through Left Antero-Lateral Thoracotomy (Thora-Cab) - A Case Report
}

\author{
Abhinav Singh ${ }^{1}$, Ravisagar Patel $^{1}$, Sarvesh Sharma ${ }^{2}$, Rajendra Mohan Mathur ${ }^{3}$ \\ ${ }^{I}$ RESIDENT M.Ch CTVS, Department of cardiothoracic and vascular surgery, Jaipur, India \\ ${ }^{2}$ RESIDENT M.Ch CTVS, Department of cardiothoracic and vascular surgery, Jaipur, India \\ ${ }^{3}$ Professor and Head of the Department of cardiothoracic and vascular surgery, Jaipur, India
}

\begin{abstract}
We report a case of 62 year old man with single vessel disease involving the left anterior descending artery (95\% proximal lesion) who underwent off-pump myocardial revascularization through antero-lateral thoracotomy (Thora--CAB). Post-operative period was uneventful with early recovery and minimal pain. He was discharged home on 5th post-operative day, doing well and is in regular follow up.

Key Words: Anterolateral thoracotomy, Coronary artery bypass grafting, Thoracab
\end{abstract}

\section{Case Report}

A 62 year old, normotensive, non diabetic gentleman, NYHA II ,with stable angina for last two years presented with gradual worsening of chest pain. Coronary angiogram showed single vessel disease involving the left anterior descending artery (95\% proximal lesion) with normal LM and RCA. Echocardiogram showed hypokinesia of middistal anterior wall, apex,mid distal IVS,distal lateral and inferior wall with LVEF $=30 \%$. The patient underwent off-pump coronary artery bypass grafting through left anterolateral thoracotomy with one lung ventilation (on double lumen ET tube) in supine position with slight right lateral tilt using $15^{\circ}$ wedge below left shoulder blade.

left anterolateral thoracotomy was performed through the left $5^{\text {th }}$ intercostals space, starting medial to the left nipple and extending to the anterior axillary line. Applying finochetto retractor, the left internal mammary artery, harvested under from its origin down to its bifurcation into the musculophrenic and superior epigastric artery. With the help of myocardial (octopus) stabilizer, distal anastomosis of Left Internal mammary artery was done to the left anterior descending artery using 7-0 prolene. Thoracotomy was closed over a thoracic drain after ensuring thorough haemostasis. Post-operatively patient was managed in in cardiac surgery ICU and discharged on $5^{\text {th }}$ post-operative day.

\section{Discussion}

Coronary revascularization on the beating heart was first proposed by Benetti and colleagues ${ }^{3}$ and Buffolo and coworkers ${ }^{4}$ for its potential benefits over conventional CABG on pump. Cardiopulmonary bypass may lead to postoperative morbidities, such as dearangement of coagulation profile, ,bleeding, fluid retention, arrhythmias, and temporary organ dysfunctions (mainly liver and renal functions ) ${ }^{1}$ In 1996, off-pump CAB was started through various means of minimal incisions, like left anterior thoracotomy and inferior median sternotomy. ${ }^{3,4,5}$ Several retrospective as well as prospective randomized studies have provided evidence for reduced morbidity associated with off-pump surgery when compared with conventional on-pump cardiopulmonary bypass. ${ }^{2}$ In particular there is evidence of reduction of inotropic requirement, arrthymias, blood loss, chest infections, sternal wound dehiscences and length of stay in hospital with early return to daily activities. The left anterolateral thoracotomy approach was chosen because potentially it offered all of the benefits of off-pump coronary revascularization, while at the same time avoiding the morbidity associated with median sternotomy. The results of studies on this approach are encouraging. It is possible to perform complete revascularization in the majority of patients. ${ }^{13}$

Patient Selection/Inclusion Criteria for thora-CAB

1.Coronary Anatomy

- Single vessel disease with normal LM and RCA

- Left main disease with normal RCA

- Triple vessel disease with medium to large PDA

- Complex proximal left sided lesions with or without large branch involvement

2.Patient with advanced age, COPD

3. Long-term steroid use or who seek out less invasive surgery options

Contraindications: Emergency cases and Patients with hemodynamic instability

Potential Contraindications: 
Previous CABG surgery, Morbid obesity, Patients with postero-lateral branch disease, Ejection Fraction $<20 \%$, Moderate to severe aortic insufficiency

In a rare event that the patient may require to be taken on cardiopulmonary bypass due to hemodynamic instability, it can be done by aortic and atrio-caval cannulation through the same approach or through the femoro-femoral route.

There have been few studies on this relatively new technique of coronary artery bypass grafting through the anterior thoracotomy approach. The most significant ones include the study by Srivastava Sudhir P. et.al. from Texas, ${ }^{5}$ Maximo Guida et.al. from Venezuela, ${ }^{6}$ and Japadman Bhaskar \&Sharma A.K. ${ }^{7}$ from New Zealand. All of them have reported good results with this technique as detailed below. Srivastava Sudhir P. et.al. have reported their experience with 200 cases from February 2000 to April 2001 with complete revascularisation through this approach using an average of 2.9 grafts per patient and have reported a mortality of $0.5 \%$ only. Only two patients required a conversion to cardiopulmonary bypass and three $(1.5 \%)$ required ventilation beyond 48hours, with no strokes throughout the study period. Maximo Guida et.al. ${ }^{6}$ have reported a series of 255 patients from November 2002 to July 2005. Complete revascularization was achieved in all patients using $3.3+1.0$ grafts. There were no conversions to cardiopulmonary bypass, and 3 patients died (1.2\%). 237 patients $(93.3 \%)$ were extubated in the operating room, and 164 patients $(65.1 \%)$ were discharged home within 48 hours after surgery. Only two patients $(0.8 \%)$ experienced a stroke. There were 14 patients $(5.5 \%)$ who experienced postoperative atrial fibrillation..

\section{Conclusion}

Coronary revascularization on the beating heart through anterolateral thoracotomy is a relatively new and acceptable technique of coronary artery bypass grafting with good results. It is a form of Minimally Invasive Direct Coronary Artery Bypass Grafting (MIDCAB) and is a step forward towards totally endoscopic coronary artery bypass grafting and robotic CABG.

\section{References}

[1]. Edmunds LH Jr. Extracorporeal Perfusion in Cardiac Surgery in the Adult. In Edmunds LH Jr, Ed. Cardiac Surgery in the Adult. McGraw-Hill, New York 1997:255-294.

[2]. Ascione R, Angelini GD. Off-pump coronary artery bypass surgery: the implications of the evidence. J Thorac Cardiovasc Surg 2003. Apr;125(4):779-781 10.1067/mtc.2003.11

[3]. Benetti FJ, Naselli G, Wood M, Geffner L. Direct myocardial revascularization without extracorporeal circulation. Experience in 700 patients. Chest 1991. Aug;100(2):312-316 10.1378/chest.100.2.312

[4]. Buffolo E, de Andrade CS, Branco JN, Teles CA, Aguiar LF, Gomes WJ. Coronary artery bypass grafting without cardiopulmonary bypass. Ann Thorac Surg 1996. Jan;61(1):63-66 10.1016/0003-4975(95)00840-3

[5]. Srivastava SP, Patel KN, Skantharaja R, Barrera R, Nanayakkara D, Srivastava V. Off-pump complete revascularization through a left lateral thoracotomy (ThoraCAB): the first 200 cases. Ann Thorac Surg 2003. Jul;76(1):46-49 10.1016/S0003-4975(03)00034-1

[6]. Guida MC, Pecora G, Bacalao Á, Muñoz G, Mendoza P, Rodríguez L. Multivessel revascularization on the beating heart by anterolateral left thoracotomy. Ann Thorac Surg 2006. Jun;81(6):2142-2146,10.1016/j.athoracsur.2006.01.054

[7]. Bhaskar J, Sharma AK. Off-Pump coronary artery revascularization via left thoracotomy -Early results. Ind J Thorac Cardiovasc Surg 2007;23:236-239.10.1007/s12055-007-0048- z 


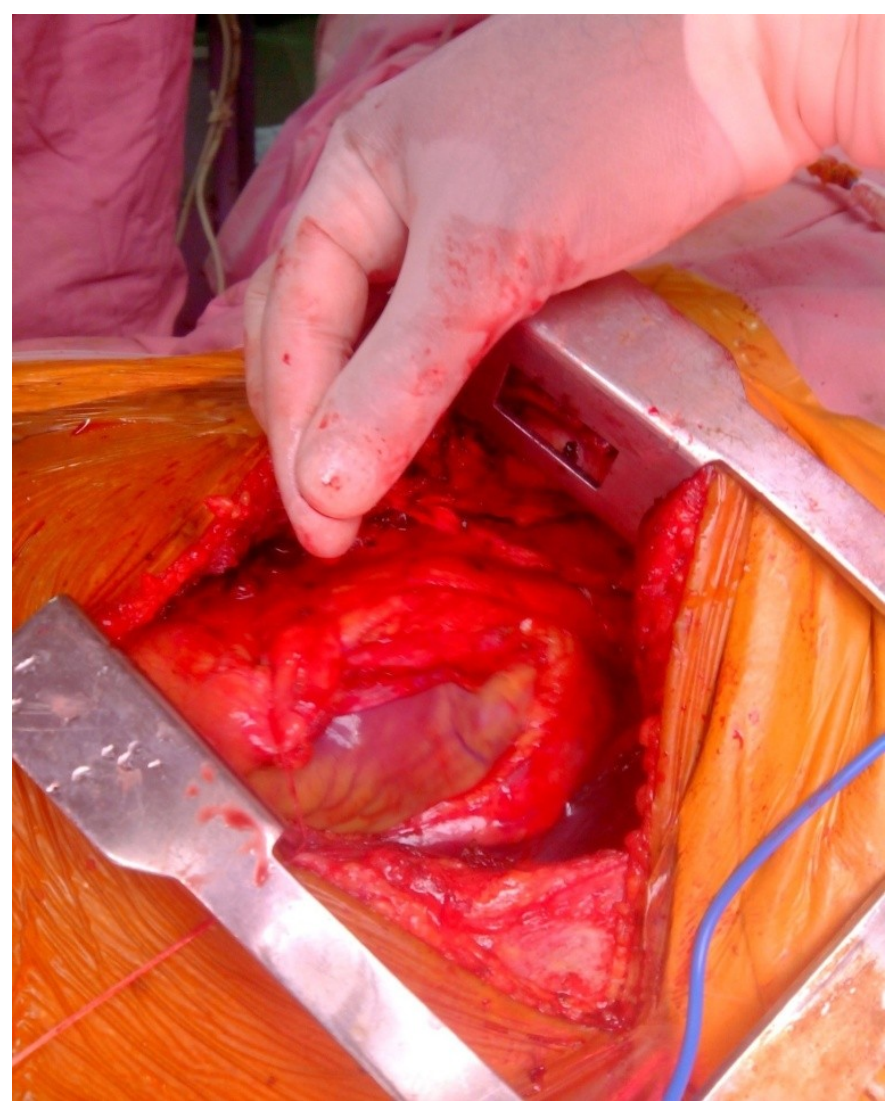

Figure 1 Operative Photograph Showing The Harvested Lefft Internal Mammary Artery.

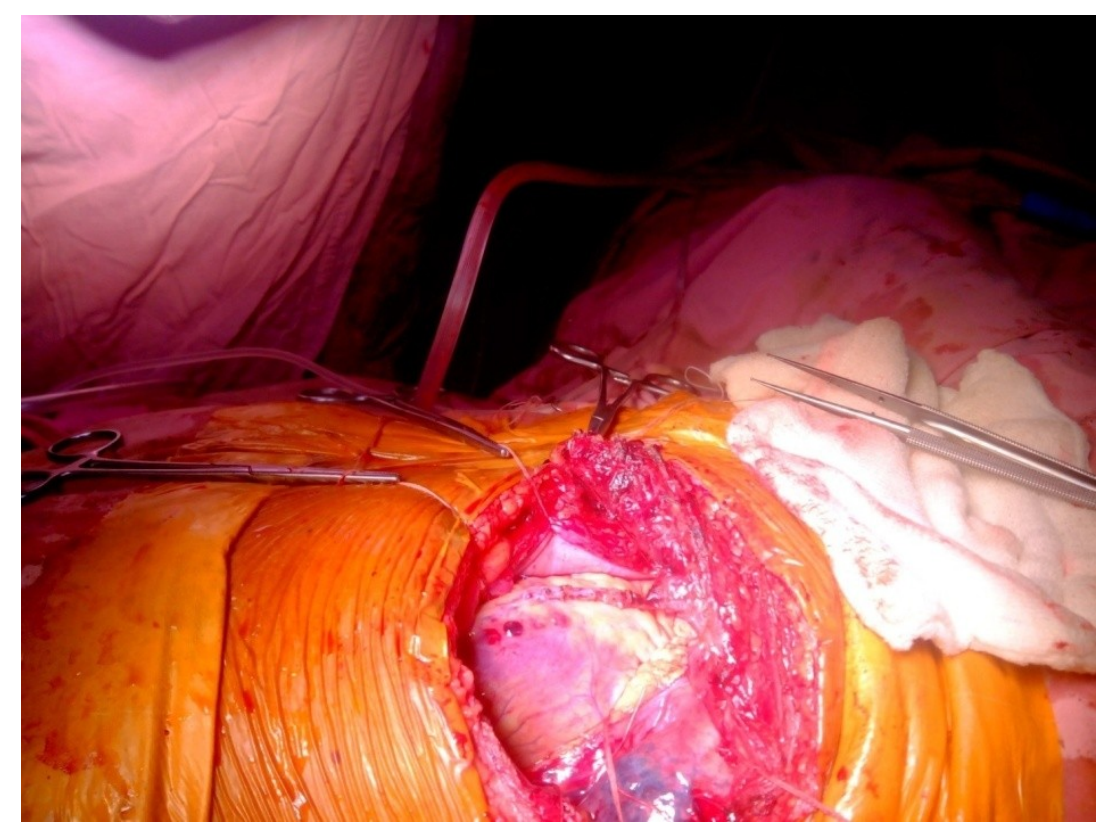

Figure 2 operative photograph showing the left internal mammary artery to left anterior descending artery anastomosis completed 